\title{
Success rate of predicting the heliospheric magnetic field polarity with Michelson Doppler Imager (MDI) synoptic charts
}

\author{
X. P. Zhao, ${ }^{1}$ J. T. Hoeksema, ${ }^{1}$ Y. Liu, ${ }^{1}$ and P. H. Scherrer ${ }^{1}$ \\ Received 16 December 2005; revised 26 May 2006; accepted 6 June 2006; published 25 October 2006.
}

[1] The photospheric and heliospheric magnetic fields have been continuously observed at the L1 point by the Michelson Doppler Imager (MDI) on the Solar and Heliospheric Observatory (SOHO) and Wind and the Advanced Composition Explorer (ACE) spacecraft since 1996. The combined observations of the photospheric and heliospheric magnetic fields make it possible to more reliably predict the heliospheric magnetic field and more accurately estimate the success rate of the prediction. On the basis of the latitude dependence of the zonal polar field inferred from high-resolution MDI synoptic charts between 1996 and 2003 we fill in the polar data gaps in the MDI synoptic charts. To assess the influence of synoptic charts from different data sources on the prediction of the coronal and heliospheric magnetic fields, we compare the success rate of the heliospheric magnetic field (HMF) polarity predicted over 107 Carrington rotations from June 1996 to June 2004. We used the potential field source surface model and the synoptic charts from the Kitt Peak National Observatory, MDI, and the Wilcox Solar Observatory. The mean success rate of the HMF polarity predicted using MDI synoptic charts over 8 years is $0.862 \pm 0.101$, the best among the three photospheric data sources, although the difference among the three sources is small. This result validates the MDI synoptic charts in modeling coronal and heliospheric magnetic fields.

Citation: Zhao, X. P., J. T. Hoeksema, Y. Liu, and P. H. Scherrer (2006), Success rate of predicting the heliospheric magnetic field polarity with Michelson Doppler Imager (MDI) synoptic charts, J. Geophys. Res., 111, A10108, doi:10.1029/2005JA011576.

\section{Introduction}

[2] The most significant feature of the heliospheric magnetic field (HMF) near the solar equatorial plane is the heliospheric current sheet (HCS) that separates the two polarity regions on the Sun and extends deep into the heliosphere. This feature has been successfully predicted and remains the major achievement among efforts to relate solar and coronal structures to heliospheric observations. The first successful prediction of the shape and location of the HCS was carried out in early 1980s using the Wilcox Solar Observatory (WSO) synoptic charts and the potential field source surface (PFSS) model [Hoeksema et al., 1982, and references therein]. With an optimum order of multipole magnetic moments, 9, and an optimum location of the source surface at 2.5 solar radii, the success rate of the prediction of the daily heliospheric magnetic field (HMF) polarity at Earth reaches $82 \%$ of days between May 1976 and January 1983 [Hoeksema, 1984]. Because the in situ observation of HMF polarity near the Earth before 1983 was not complete, the polarity inferred from geomagnetic data was used to fill data gaps. This kind of replacement may affect the reliability of the success rate estimated.

\footnotetext{
${ }^{1}$ W. W. Hansen Experimental Physics Laboratory, Stanford University, Stanford, California, USA

Copyright 2006 by the American Geophysical Union. 0148-0227/06/2005JA011576\$09.00
}

[3] There have been attempts to increase the success rate of the prediction by improving the coronal field models. The first attempt, the PFSS model with a radial boundary condition [Wang and Sheeley, 1992; Zhao and Hoeksema, 1993], assumes that the photospheric magnetic field is purely radial everywhere on the photosphere. The measured line-of-sight component can thus be easily converted to the radial field, and the radial field can be matched by the radial component of the potential field. The next improvement is the current sheet (CS) model similar to Schatten's [1971] model, which includes the effect of the HCS on the field above the cusp points [Zhao and Hoeksema, 1994; Wang, 1996]. Another significant alternative for modeling the coronal field is to solve the three-dimensional magnetohydrodynamic (MHD) equations to determine the time-varying state of the coronal magnetic field and of coronal plasma. In these calculations, the time-dependent MHD equations are solved until a steady state is established [Mikic and Linker, 1996]. In the MHD model, the observed photospheric magnetic field is applied as a boundary condition. The plasma density and temperature on the photosphere are usually assumed to be uniform. For computational reasons, the MHD model must usually be based on a coarser grid at the inner boundary than the PFSS model.

[4] In the meantime significant progress in observations of the solar and heliospheric magnetic fields have been made since 1996. Both the photospheric and heliospheric magnetic fields can be continuously measured at the L1 point by the $\mathrm{MDI} / \mathrm{SOHO}$, the Wind, and the ACE. The complete set of 
a

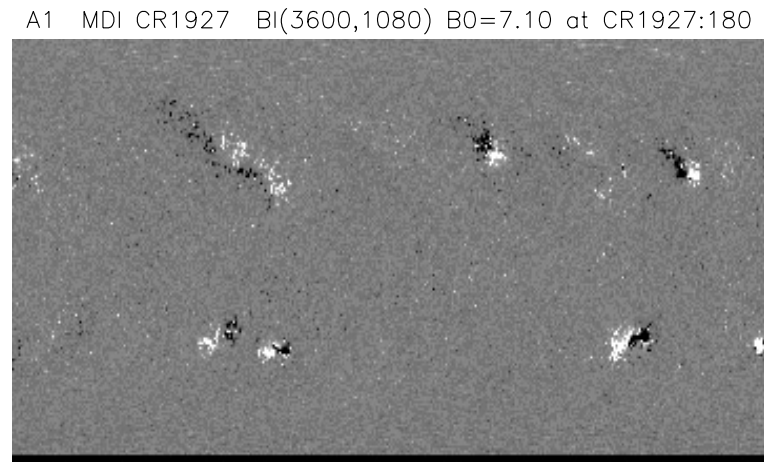

C

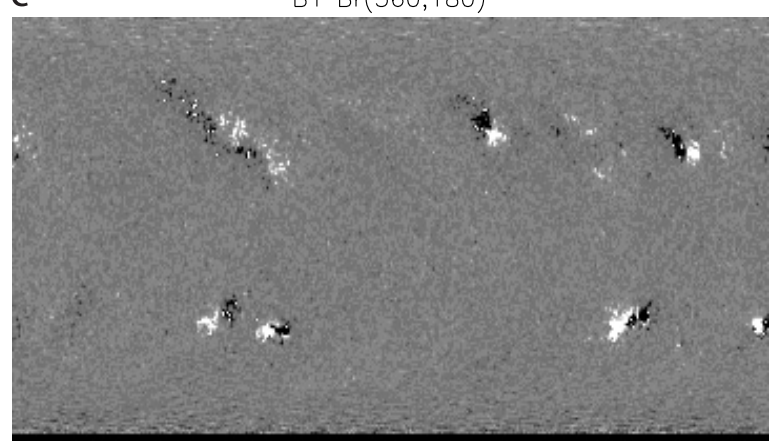

e

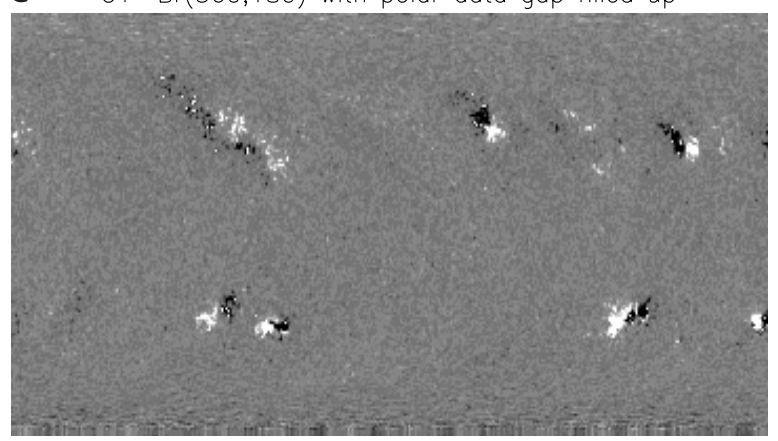

b

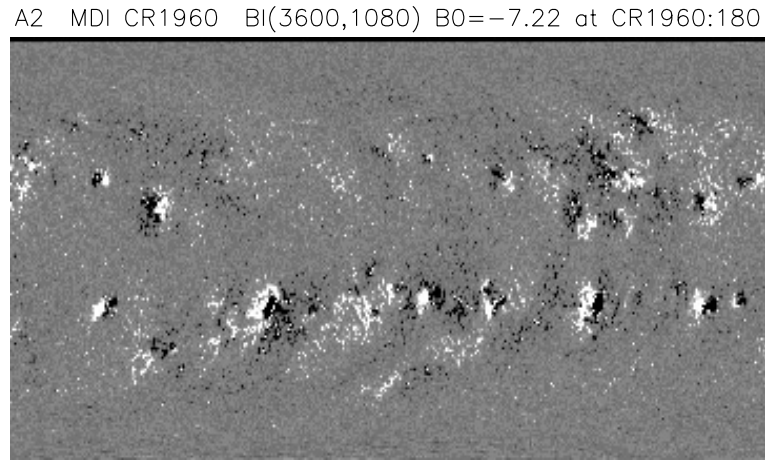

d B2 $\operatorname{Br}(360,180)$

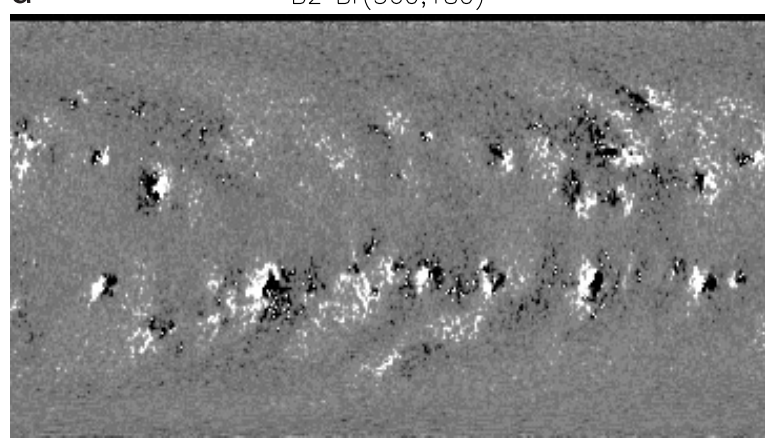

f

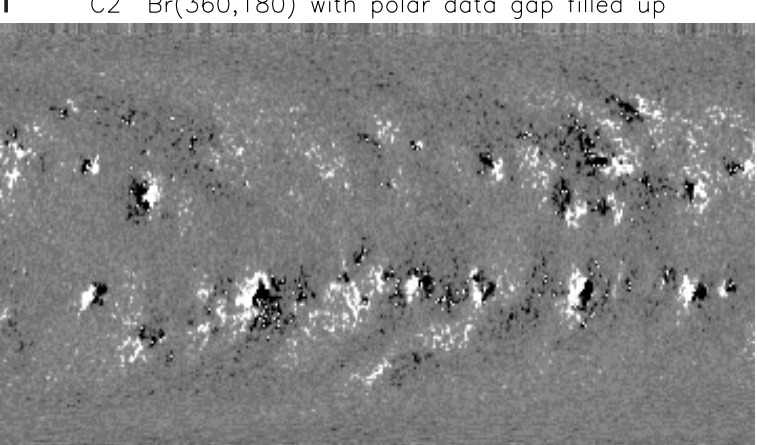

Figure 1. Maps showing $360^{\circ}$ of longitude and from -1 to 1 in sine latitude. Positive and negative field areas are shown as white and black. The grid spacing is shown on the top of each panel. (a and b) Lineof-sight component in high resolution and (c and d) radial component in lower resolution. The black zones in Figures 1a-1d denote data gaps in polar regions that depend on the $B_{0}$ angle and need to be filled in. (e and f) Data gap filled in.

observations of the photospheric magnetic field can be used to construct the MDI synoptic charts that more nearly approximate the whole surface distribution of the photospheric field. The comprehensive measurements of the HMF near the Earth make the assessment of a success rate more reliable.

[5] Neugebauer et al. [1998] compared observations during Ulysses's first rapid sweep through $>90^{\circ}$ of solar latitude in early 1995 with the latitudinal extent of the HCS predicted using various models, i.e., the PFSS models with line-of-sight and radial boundary conditions, the CS model [Wang, 1996], and the MHD model. The synoptic charts of the photospheric magnetic field used in the calculations were constructed using magnetograms observed at WSO and the Kitt Peak National Observatory (KPNO). Use of the two different data sources results in latitudinal differences of the predicted location of the HCS less than or equal to $10^{\circ}$; there is up to $21^{\circ}$ spread in latitude for the HCS positions calculated from these models, though the HCS crossing in longitude for these model is basically identical.

[6] To see if the new MDI synoptic charts lead to any improvement in predicting the HCS crossing, this work calculates the HMF polarity using the MDI, KPNO, and WSO synoptic charts and the PFSS model with the radial boundary condition. We compare the predicted HMF polarity with the HMF polarity observed by the spacecrafts Wind and ACE from June 1996 to June 2004. The paper first describes how to fill the data gaps in the polar regions of the MDI synoptic charts published in SOI Web site; we then calculate the location of HCS between Carrington rotation (CR) 1911 

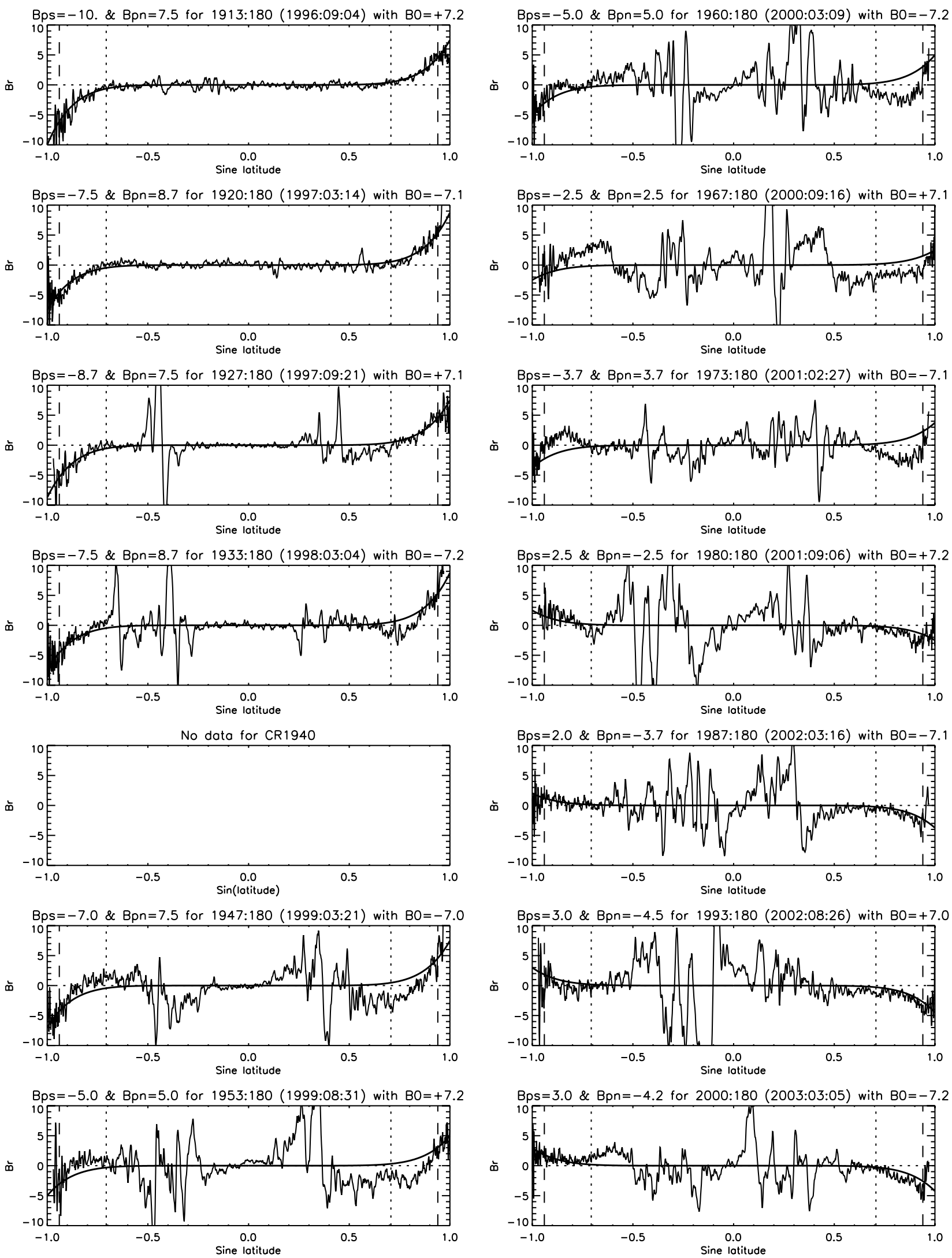

Figure 2. Sine latitude dependence of the longitude-averaged field for Carrington rotations with highest $B_{0}$ angle between September 1996 and March 2003. The thick fitting lines are produced by expressions $B_{r}=B_{p n} \sin ^{8} \theta\left(B_{r}=B_{p s} \sin ^{8} \theta\right)$ for north (south) polar region within each panel. The values of $B_{p n}$ and $B_{p s}$ in Gauss are shown on the top of each panel. The dotted and dashed vertical lines denote sine latitude when latitudes equal $\pm 45^{\circ}$ and $\pm 70^{\circ}$. 
and 2017 and compare the success rate of the prediction of the daily HMF polarity; finally we summarize and discuss the results.

\section{MDI Synoptic Charts}

[7] MDI synoptic charts published in the SOI Web page $\mathrm{http} / / /$ soi.stanford.edu/magnetic/index6.html) are the proxies of entire surface distributions of line-of-sight components of the photospheric magnetic field. The MDI synoptic charts preserve the spatial resolution of the observed MDI magnetic images. Their grid is 3600 pixels in longitude and 1080 pixels in sine latitude. These high resolution synoptic charts of photospheric magnetic fields can be used to examine the magnetic characteristics in small-scale features observed in other spectral lines. Figures $1 \mathrm{a}$ and $1 \mathrm{~b}$ show the highresolution MDI synoptic charts for CR1927 and CR1960 when the $B_{0}$ angle, the heliographic latitude of the Earth, is highest and the sunspot number is near the minimum and maximum of solar cycle 23, respectively. During 1997:09:21 and 2000:03:09 corresponding to CR1927:180 and CR1960:180, the Earth was located at $7.10^{\circ}$ and $-7.22^{\circ}$ from the solar equator, as shown on the top of each panel by $B_{0}$ angle, and the data gap occurred in south and north polar regions, respectively, as shown by black zones. The data gaps for the two solar rotations occur at north or south latitudes higher than $76^{\circ}$ or at sine latitudes higher than 0.97 .

[8] As mentioned in section 1, the photospheric field, especially the polar field, may be assumed to be purely radial, so that the radial field strength can be inferred from the lineof-sight measurement. It is well known that the coronal and heliospheric fields are determined by the large-scale photospheric field. The high-resolution synoptic chart can be reduced to lower spatial resolution using nearest neighborhood averaging. Figures $1 \mathrm{c}$ and $1 \mathrm{~d}$ show the lower resolution $(360 \times 180)$ synoptic charts of the radial field. The corresponding data gaps for the two rotations occur at north or south latitudes higher than $73^{\circ}$ or at sine latitudes more than 0.95 .

[9] It has been shown [Hoeksema, 1984, and references therein] that polar fields are one of the most sensitive factors that determine the shape of the HCS. Thus correctly filling up the polar data gaps in MDI synoptic charts is a key step to successfully extrapolating the photospheric field observed by MDI into the corona and heliosphere.

[10] The polar fields observed in different observatories all show an annual variation, suggesting that the polar magnetic field is radially directed and it increases as the latitude increases [Svalgaard et al., 1978]. For WSO data in 1976-1977, it has been shown that the latitudinal variation of the radial polar field follows

$$
B_{r}=B_{p} \sin ^{n} \theta
$$

where $B_{p}$ denotes the field strength at the pole, $\theta$ is the latitude, and $n$ equals 8 or 7 [Svalgaard et al., 1978; Wang and Sheeley, 1995].

[11] Figure 2 displays the latitude dependence of the zonal average of the radial field strength obtained from MDI highresolution synoptic charts for those Carrington rotations between September 1996 and March 2003 that have the highest $B_{0}$ angle. The fluctuating curve in each panel shows the zonal average from south to north poles and the smoothed thick curve that fits the fluctuating curve is obtained using expression (1) with $n=8$ and $B_{p}$ for north and south poles, $B_{p n}$ and $B_{p s}$, shown on the top of each panel. The dotted and dashed vertical lines denote, respectively, latitudes of $\pm 45^{\circ}$ and $\pm 70^{\circ}$. Figure 2 shows that the polar field above latitude of $73^{\circ}$ or sine latitude of 0.95 can be fitted grossly by $\sin ^{8} \theta$; in the years of 1996, 1997, 1998 and 2002, 2003, the fitting is very good, confirming the early finding from WSO data. It should be noted that the difference between $\sin ^{8} \theta$ and $\sin ^{7} \theta$ is only 0.01 when $\theta>80^{\circ}$.

[12] We approximately estimate individual $B_{p}$ using the nearest neighborhood averaged $B_{r}$ at the points adjacent to the pixel where no data is observed. For instance, there is a data gap between a pole and a given pixel with latitude of $76^{\circ}$ and longitude of $180^{\circ}$. We first find out an adjacent pixel that has longitude of $180^{\circ}$ and latitude lower than but adjacent to $76^{\circ}$, and then obtain a specific $B_{p}$ using the nearest neighborhood averaged $B_{r}$ at the adjacent pixel on the basis of expression (1). It should be emphasized that the two poles are the geometrically singular point in the format of synoptic charts, and $B_{p}$ at the poles could not be uniformly specified. Figures $1 \mathrm{e}$ and $1 \mathrm{f}$ show the result. In following calculations we use the grid of $360 \times 180$ for MDI and KPNO synoptic charts and the grid of $72 \times 30$ for WSO synoptic chart.

\section{Comparison of Predicted and Observed HMF Polarities}

[13] Neugebauer et al. [1998] have shown that the PFSS model is in close agreement with the MHD model for predicting the HCS. We extrapolate the entire surface distribution of the photospheric magnetic field into the corona using the PFSS model with the radial boundary condition.

[14] There are two free parameters in the PFSS model: the principal order of the spherical harmonic series, $N$, and the heliocentric distance of the source surface, $R_{S s}$. Trial and error has been used for WSO synoptic charts to find the optimum $N$ and $R_{s s}$ that make the predicted HCS crossings best agreeing with the observations [Hoeksema, 1984]. A pair of optimum parameters, $N=9$ and $R_{S S}=2.5$ solar radii, have been widely used in the literature since then. The spherical harmonic series is obtained by decomposing the entire surface distribution of the photospheric field. The optimum $N$ determined using MDI synoptic charts may thus be different from using WSO synoptic charts.

[15] To assess the influence of different data sources, i.e., synoptic charts of MDI, KPNO and WSO, on the prediction of the HCS, we first examine the effect of the spatial resolution of the synoptic charts on the calculated source surface field since the grid spacing for MDI and KPNO synoptic charts is $360 \times 180$, and for WSO synoptic charts it is $72 \times 30$. Figures $3 a, 3 b, 3 e$, and 3 fare MDI synoptic charts with grid spacing of $360 \times 180$ and $72 \times 30$, respectively, and Figures $3 \mathrm{c}, 3 \mathrm{~d}, 3 \mathrm{~g}$, and $3 \mathrm{f}$ are the source surface fields calculated using spherical harmonic coefficients obtained from the synoptic charts of Figures $3 \mathrm{a}, 3 \mathrm{~b}, 3 \mathrm{e}$, and $3 \mathrm{f}$. The very high correlation coefficients (see "CC" in Figures $3 \mathrm{~g}$ and $3 \mathrm{~h}$ ) between the source surface fields in second and fourth rows imply that both in low and high solar activities, the effect of spatial resolution of synoptic charts on the source surface field can be neglected. 
a
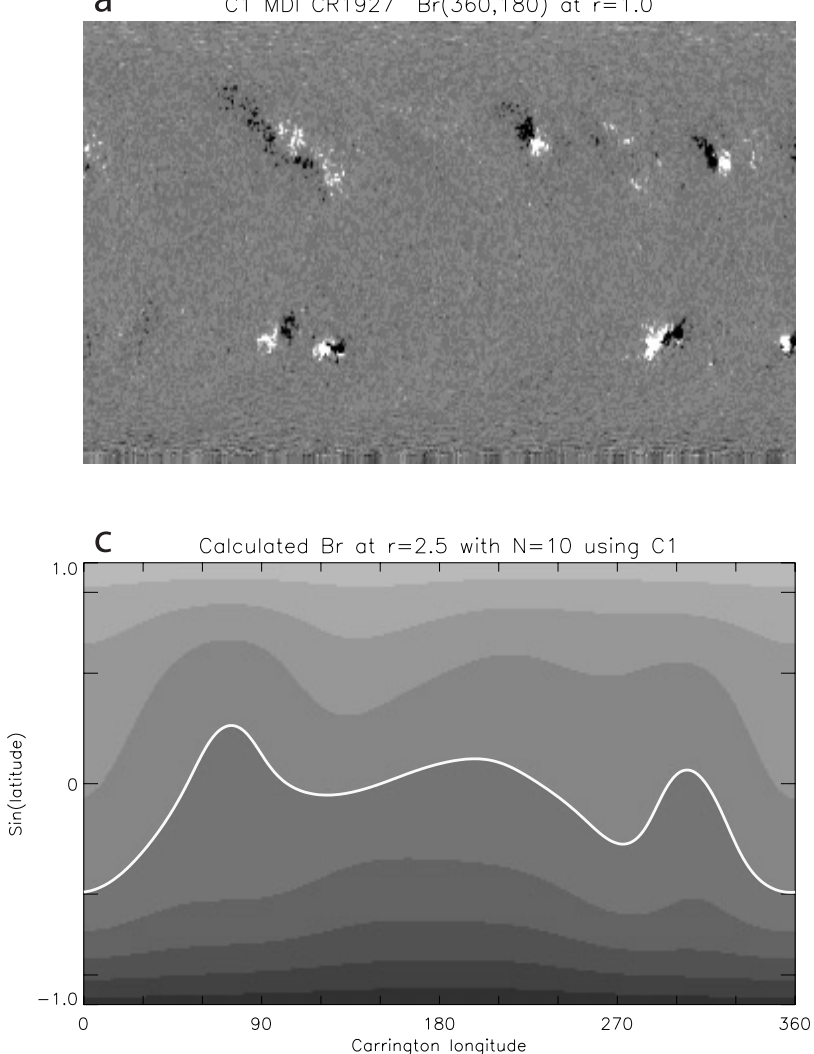

e D1 MDI CR1927 $\operatorname{Br}(72,30)$ at $r=1.0$
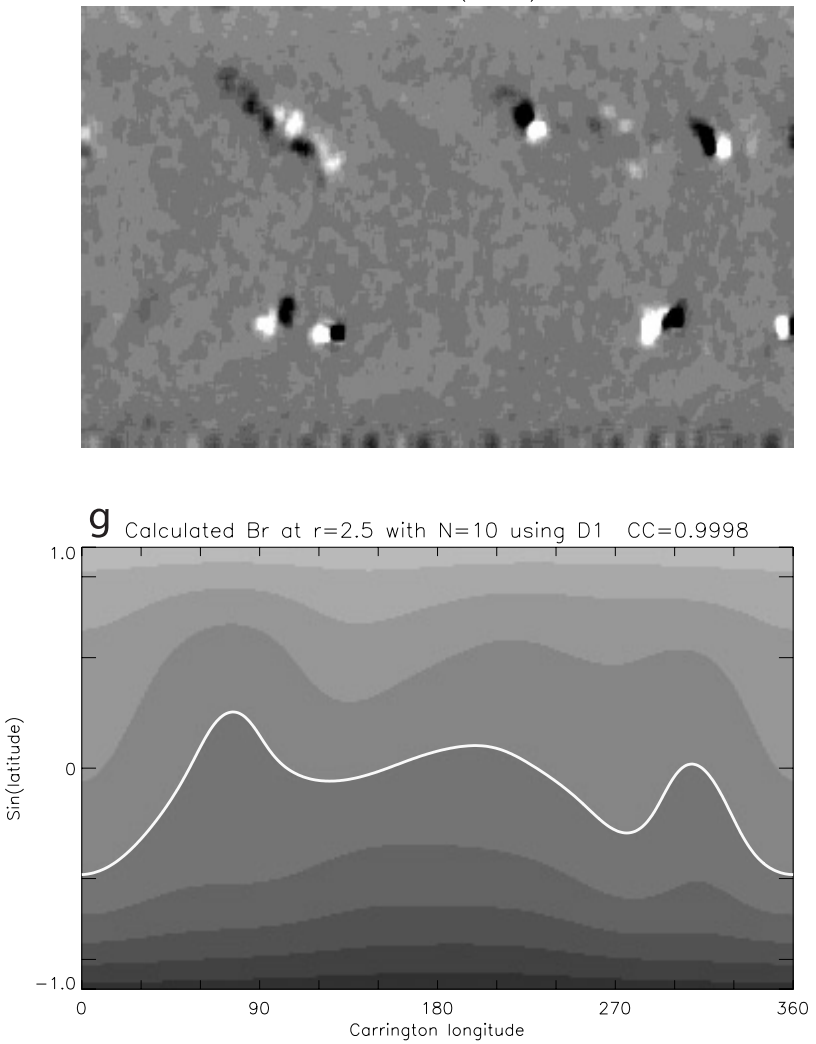

b
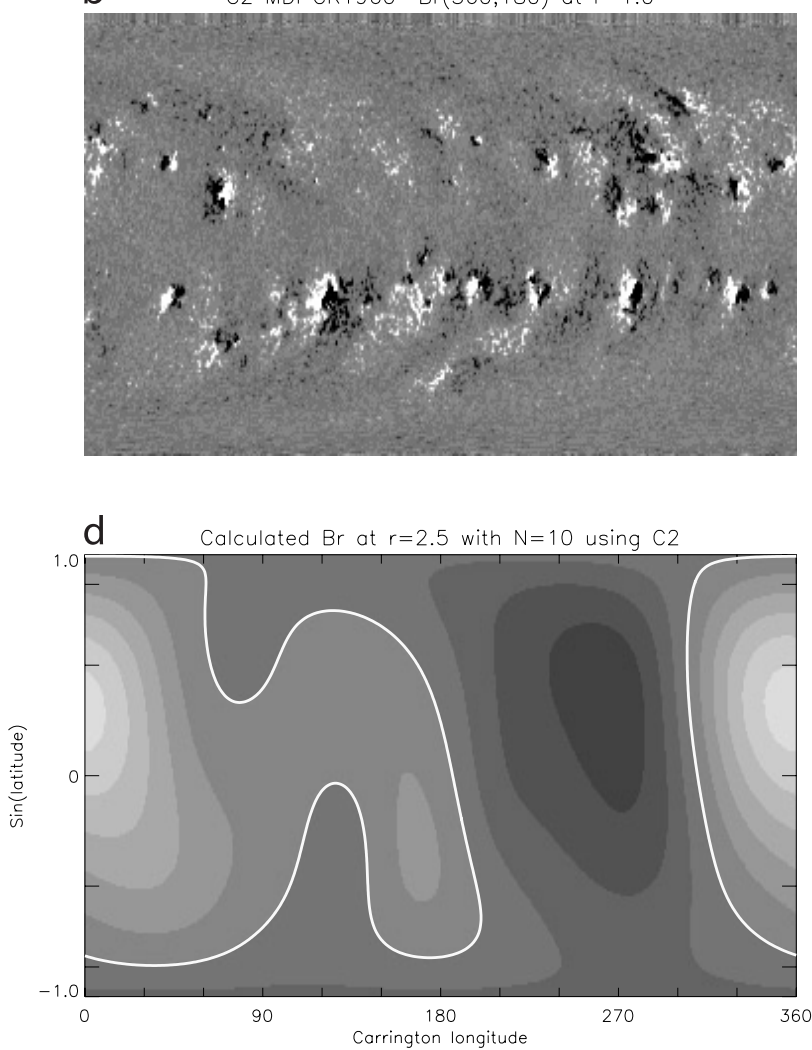

f

D2 MDI CR1960 $\operatorname{Br}(72,30)$ at $r=1.0$
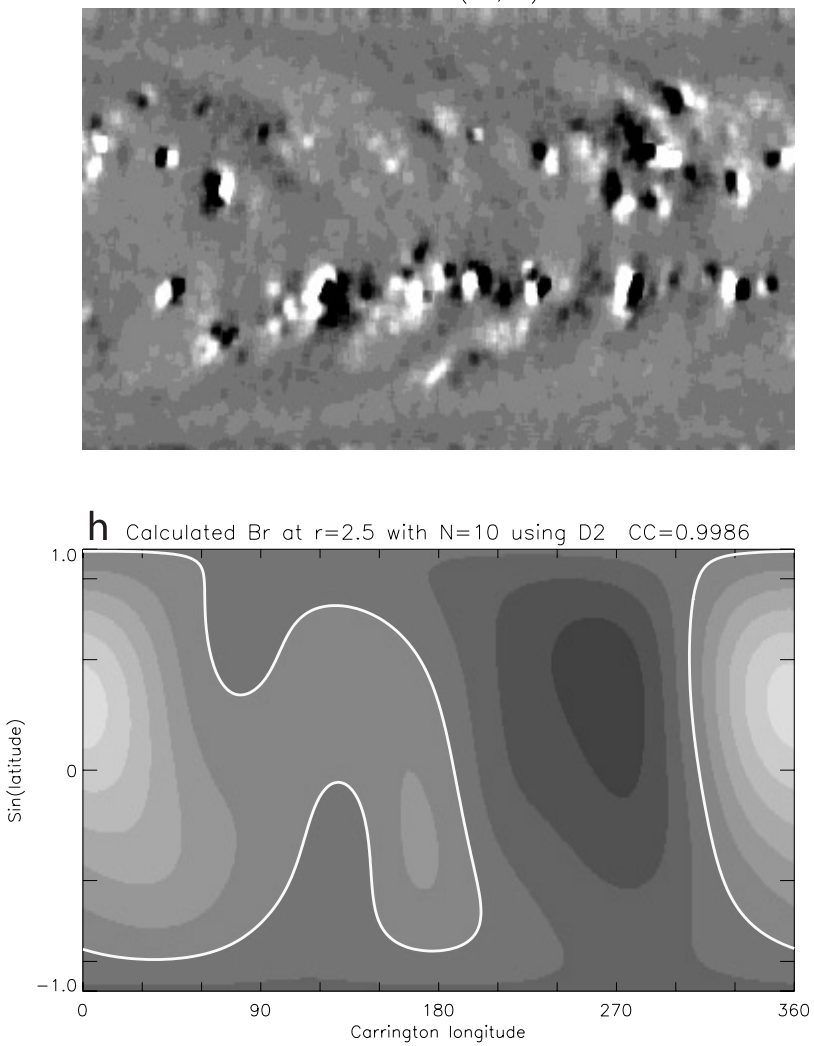

Figure 3. Comparison of the source surface field calculated using $(a-d)$ MDI $360 \times 180$ synoptic charts with $(\mathrm{e}-\mathrm{h})$ that using MDI $72 \times 30$ synoptic charts. "CC" denotes the correlation coefficients between the source surface field calculated using synoptic charts with different spatial resolution. The white line in the source surface plots is the neutral line separating positive and negative polarity regions (lighter and darker shading). 

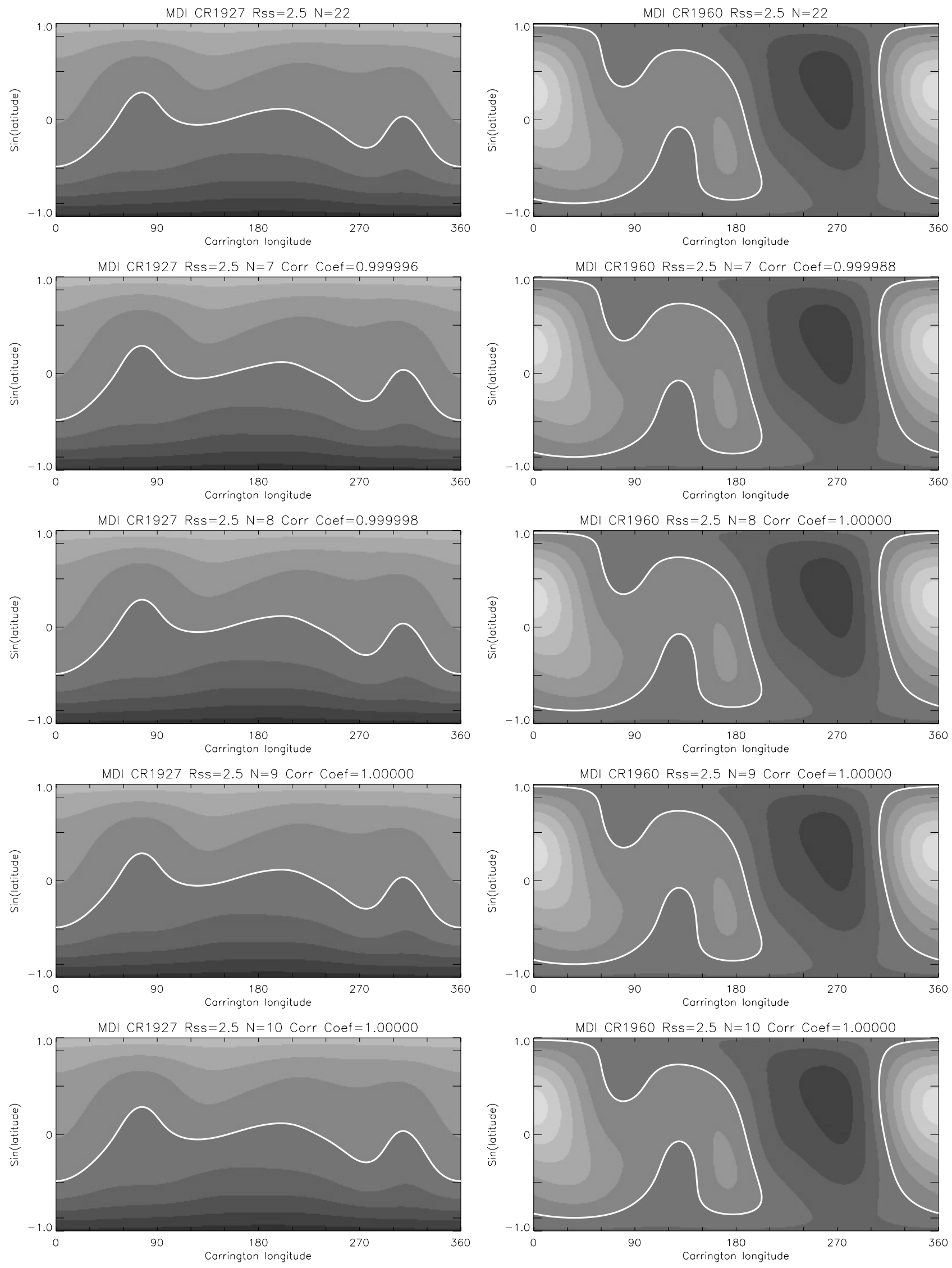

Figure 4. Source surface field calculated using various $N$, as shown on the top of each panel, for CR1927 and CR1960 and their correlation coefficients with the source surface fields calculated using $N=$ 22 (see the top of each panel). 
Table 1. Optimum $N$ for Each Observatory

\begin{tabular}{lcc}
\hline & CR1927 & CR1960 \\
\hline KPNO & 9 & 8 \\
MDI & 9 & 8 \\
WSO & 9 & 10 \\
\hline
\end{tabular}

[16] We use a new method to determine an optimum $N$ that corresponds to a specific $R_{s s}$ and is valid for all three data sources. It is generally thought that the representation of the entire surface distribution of the photospheric field by the spherical harmonic series is the better, the greater the value of $N$. We found recently, however, that the photospheric field calculated with the principal number $N=22$ has the highest correlation coefficient with the synoptic charts that have the same spatial resolution as WSO [Poduval and Zhao, 2004]. This result may be understood by the "Gibbs's phenomenon" due to the discontinuity at longitudes of $0^{\circ}$ and $360^{\circ}$ [Chapman and Bartels, 1962].

[17] As the heliocentric distance, $r$, increases, the amplitude of multipole components decreases as $r^{-(N+2)}$. Thus the optimum $N$ for calculating the source surface field at $R_{S S}=2.5$ solar radii must be less than 22 for KPNO, MDI and WSO synoptic charts. The top row of Figure 4 displays the field distribution on the source surface of $R_{s s}=2.5$ calculated using $N=22$ and the MDI synoptic charts of CR1927 and CR1960, as shown by Figures 1e and 1f. The other rows in Figure 4 show the calculated source surface field using different $N$ values as shown on the top of each panel. Also shown on the top of each panel is the correlation coefficient of the calculated source surface field with the field in the top row of Figure 4. For MDI synoptic charts of CR1927 (CR1960), the source surface field calculated using $N$ greater than $9(8)$, is exactly the same as that using $N=22$. Thus we may select $N=9$ as the optimum $N$ for MDI synoptic charts both in minimum and maximum phases. Table 1 shows the optimum $N$ for KPNO, MDI, and WSO synoptic charts of CR1927 and CR1960. Thus we select $N=10$ as the optimum $N$ for calculating the location of HCSs using all three data sources.

[18] In the following calculations we calculate the location of HCSs using $N=10$ and $R_{s S}=2.5$ solar radii for synoptic charts of KPNO, MDI and WSO from CR1911 to CR 2017 between June 1996 and June 2004 when daily HMF polarity observed by Wind and ACE can be obtained from the OMNI Web site http://nssdc.gsfc.nasa.gov/omniweb).

[19] Figure 5 shows the shape of HCSs calculated using the three data sources. The difference in the Carrington rotation number is 13 between adjacent panels. In each panel, three contours are displayed, corresponding to $-0.1,0.0$, and 0.1 Gauss. All pixels located in the $0.1(-0.1)$ side of the 0 contour are in positive (negative) polarity of the predicted HMF. Since the solar wind speed around sector boundaries is systematically low, we map the HCS from 2.5 solar radii to $1 \mathrm{AU}$ with a speed of $350 \mathrm{~km} / \mathrm{s}$ [Borrini et al., 1981] to compare the predicted HMF polarity with the observed. The Carrington longitude correspondingly is left shifted by 55 degrees from 360 to 295 at both sides of each solar rotation. The shape of the calculated HCSs in Figure 5 looks similar among the three data sources for most of Carrington Rotation numbers.
[20] To compare the predicted HMF polarity with that observed at the Earth for each solar rotation, we first pick up the observed daily HMF polarities occurred between the starting and ending dates of each rotation. For example, the starting and ending dates for CR1911 are 3 July 1996 and 30 July 1996, corresponding to CR1911:295 and CR1912:295 (see the middle panel of the top row of Figure 5), respectively. The daily HMF polarity is determined on the basis of azimuthal angles of daily HMF with values in between $135^{\circ} \pm 30^{\circ}$ for the direction away from the Sun and in between $315^{\circ} \pm 30^{\circ}$ for the direction toward the Sun. The range of $60^{\circ}$ is used in identifying the HMF polarity for ensuring that it is the polarity of ambient HMF. The plus and minus symbols in each panel of Figure 5 denote such identified positive and negative HMF polarities. For CR1911, we found 24 observed daily ambient HMF polarities. The success rate of the prediction may be visually estimated by comparing the observed HMF polarities with their corresponding ones located in both sides of the predicted HCS. Since most of the discrepancies occur in the day or two near the HCS crossing, the inaccuracy of the solar wind speed that is used to map the the predicted HCS to the Earth may be the major factor to determine the success rate. To more accurately determine the success rate of the prediction, we map the $B_{0}$ angle and the Carrington longitude for each date when the daily HMF polarity was observed back to 2.5 solar radii using the observed daily solar wind speed, and calculate the source surface field polarity at this mapped point. The success rate indicated in each panel denotes the rate of the number of predicted HMF polarities that agree with observed daily HMF polarities to the total selected number for each rotation.

[21] Figure 6 shows the success rate of the prediction of the HMF polarity for each Carrington rotation, for 12-rotation average and for the mean values of the whole data set. The curves with colors of red, green and blue denote the rotation success rate for MDI, WSO and KPNO data sources. The dots with three colors denote the averaged success rate over 12 Carrington rotations. The mean success rate and standard deviation for all 107 synoptic charts from MDI, KPNO and WSO are $0.862 \pm 0.101,0.855 \pm 0.115$, and $0.845 \pm 0.127$, as shown in Figure 6 as the dashed and dotted lines.

\section{Summary}

[22] We have shown using high-resolution MDI synoptic charts that as solar latitude, $\theta$, increases, the zonal polar field above latitude of $73^{\circ}$ increases as $\sin ^{8} \theta$. By using this latitude dependence of the polar field we fill data gaps in MDI synoptic charts.

[23] We develop a method to find the optimum $N=10$ that is valid for all three data sources, i.e., KPNO, MDI and WSO synoptic charts.

[24] The mean success rates of the prediction of the daily HMF polarity over 8 years are $85.5 \% \pm 11.5 \%, 86.2 \% \pm$ $10.1 \%$, and $84.5 \% \pm 12.7 \%$ for KPNO, MDI, and WSO, respectively. Considering that this is a prediction using photospheric data and that most of the discrepancies occur in the day or two near the HCS crossings, all predictions for the three data sources are excellent. Among the three photospheric data sources, the MDI synoptic charts produce the 

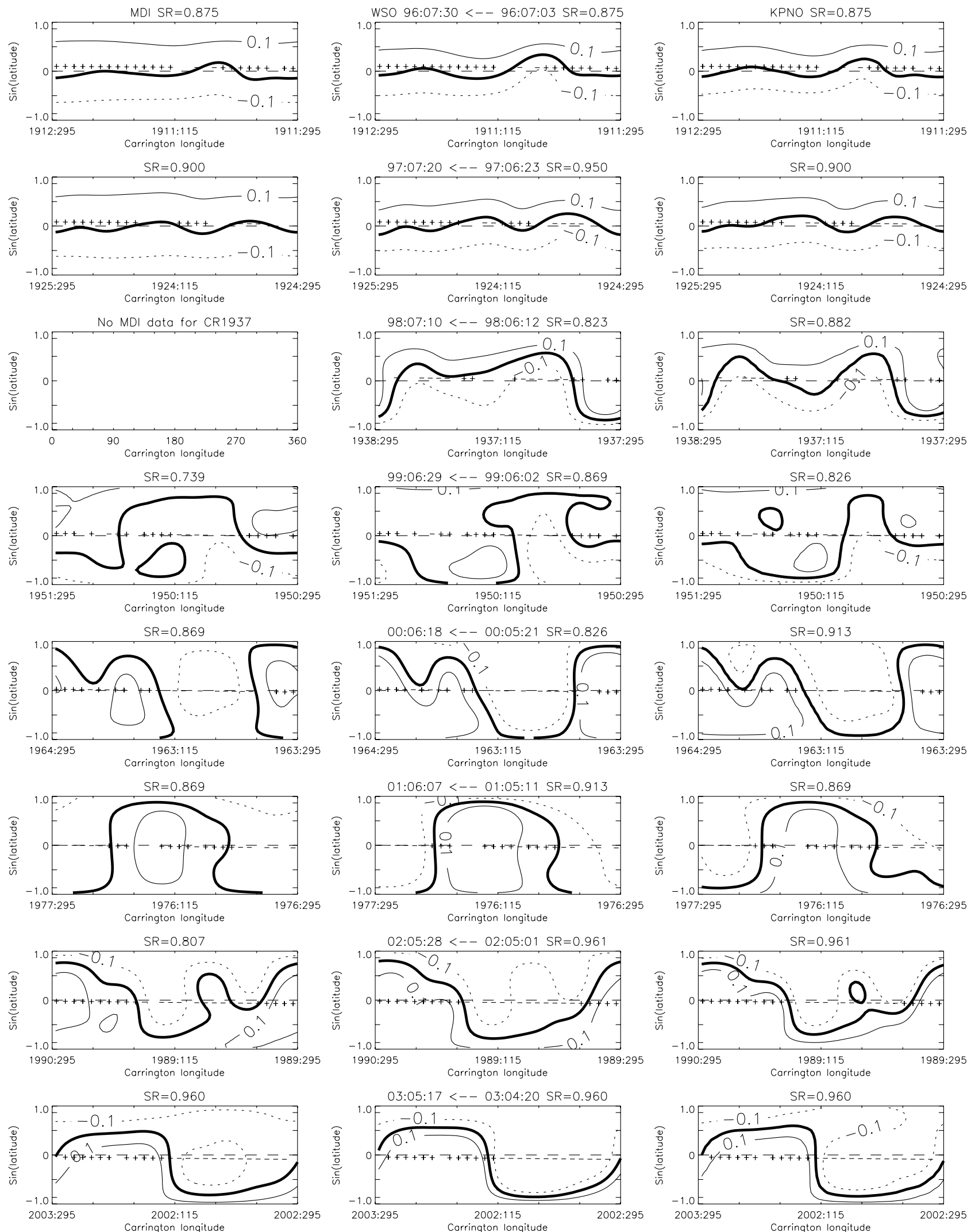

Figure 5. Calculated three contours corresponding to $-0.1,0.0$, and 0.1 Gauss at the source surface for each solar rotation. The thick contour denotes the HCS. Pluses and minuses denote positive and negative HMF polarity observed near the Earth. The left, middle, and right columns are obtained using MDI, WSO, and KPNO photospheric data sources. The Carrington longitudes correspond to the time when the HMF polarities were observed near the Earth. "SR" denotes the success rate of the prediction. 


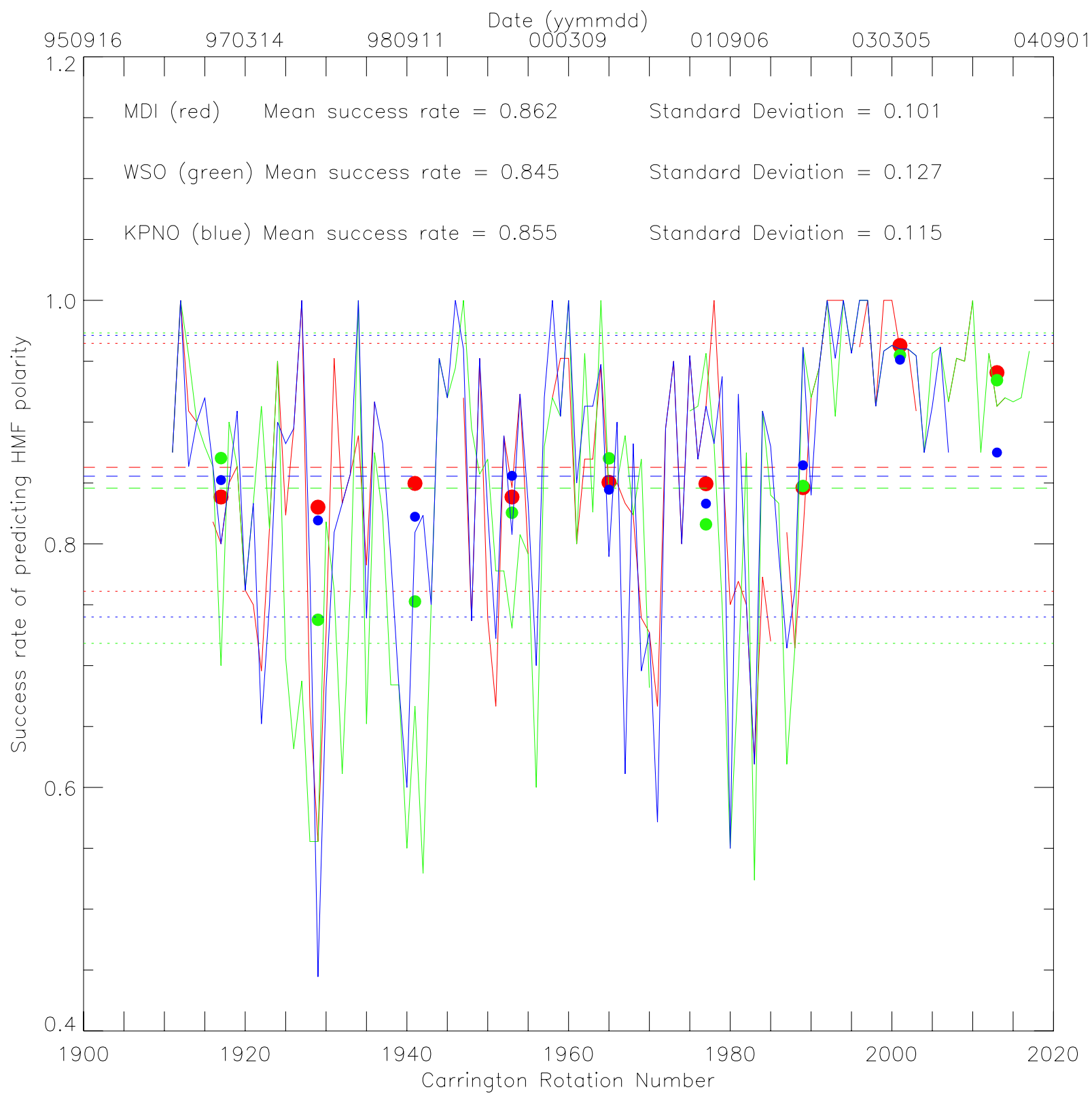

Figure 6. Success rate of the prediction of the HMF polarity for each rotation (solid lines), 12-rotation average (dots), and 107-rotation average (dashed lines). The dotted lines show the standard deviation with respect to the 107-rotation average. Red, green, and blue show MDI, WSO, and KPNO, respectively.

highest mean success rate and the lowest standard deviation, though the differences among the three sources are small. The result implies that the MDI synoptic chart constructed using the complete MDI images over a solar rotation is certainly usable and useful, if not the best, in coronal and heliospheric modeling.

[25] It should be noted that a comparison of the HMF polarity predicted with HMF polarities observed at Earth is only a weak test in validating the synoptic chart and coronal field model. The work in comparing the HMF strength as well as the HMF polarity using CSSS model and the MDI synoptic chart is in progress and expected to be better than PFSS models.

[26] Acknowledgments. The KPNO data and the Wind and ACE data are downloaded from the KPNO and OMNI Web sites, respectively. This work was supported by NASA grants NAGW 2502 and NAG5-3077 and by NSF grant ATM9400298.

[27] Amitava Bhattacharjee thanks Natalie Krivova for her assistance in evaluating this paper.

\section{References}

Borrini, G., J. T. Gosling, S. J. Bame, W. C. Feldman, and J. M. Wilcox (1981), Solar wind helium and hydrogen structure near the heliospheric current sheet: A signal of coronal streamers at 1 A.U., J. Geophys. Res., $86,4565$.

Chapman, S., and J. Bartels (1962), Geomagnetism, vol. II, Oxford Univ. Press, New York.

Hoeksema, J. T. (1984), Structure and evolution of the large scale solar and heliospheric magnetic fields, Ph.D. thesis, Stanford Univ., Stanford, Calif. Hoeksema, J. T., J. M. Wilcox, and P. H. Scherrer (1982), Structure of the heliospheric current sheet in the early portion of sunspot cycle 21, J. Geophys. Res., 87, 10,331.

Mikic, Z., and J. A. Linker (1996), The large-scale structure of the solar corona and inner heliosphere, in Solar Wind Eight, edited by D. Winterhalter et al., AIP Proc., 382, 104. 
Neugebauer, M., et al. (1998), Spatial structure of the solar wind and comparisons with solar data and models, J. Geophys. Res., 103, 14,587.

Poduval, B., and X. P. Zhao (2004), Discrepancies in the prediction of solar wind using potential field source surface model: An investigation of possible sources, J. Geophys. Res., 109, A08102, doi:10.1029/2004JA010384.

Schatten, K. H. (1971), Current sheet magnetic model for the solar corona, Cosmic Electrodyn., 2, 232.

Svalgaard, L., T. L. Duvall Jr., and P. H. Scherrer (1978), The strength of the Sun's polar fields, Sol. Phys., 58, 225.

Wang, Y.-M. (1996), Nonradial coronal streamers, Astrophys. J., 456, L119.

Wang, Y.-M., and N. R. Sheeley Jr. (1992), On potential field models of the solar corona, Astrophys. J., 392, 310.

Wang, Y.-M., and N. R. Sheeley Jr. (1995), Solar implications of Ulysses interplanetary field measurements, Astrophys. J., 447, L143.
Zhao, X. P., and J. T. Hoeksema (1993), Unique determination of model coronal magnetic fields using photospheric observations, Sol. Phys., 143, 41.

Zhao, X. P., and J. T. Hoeksema (1994), A coronal magnetic field model with horizontal volume and sheet currents, Sol. Phys., 151, 91.

J. T. Hoeksema, Y. Liu, P. H. Scherrer, and X. P. Zhao, W. W. Hansen Experimental Physics Laboratory, Stanford University, Stanford, CA 94305-4085, USA. (hthoeksema@solar.stanford.edu; yliu@solar.stanford. edu; phscherrer@solar.stanford.edu; xuepu@quake.stanford.edu) 